\title{
Semitransparent organic photovoltaic cells
}

\author{
Rhonda F. Bailey-Salzman and Barry P. Rand \\ Department of Electrical Engineering, Princeton University, Princeton, New Jersey 08544 \\ Stephen R. Forrest ${ }^{\text {a) }}$ \\ Department of Electrical Engineering and Computer Science, University of Michigan, Ann Arbor, Michigan \\ 48109; Department of Physics, University of Michigan, Ann Arbor, Michigan 48109; \\ and Department of Materials Science and Engineering, University of Michigan, Ann Arbor, \\ Michigan 48109
}

(Received 25 February 2006; accepted 8 May 2006; published online 5 June 2006)

\begin{abstract}
We demonstrate semitransparent, small molecular weight organic solar cells employing a thin silver/indium tin oxide compound cathode with a maximum transmission of $(60 \pm 6) \%$ averaged over the visible spectral range and with a power conversion efficiency, $\eta_{p}=(0.28 \pm 0.03) \%$ under simulated, AM1.5G, 1 sun illumination. By increasing the Ag thickness, an average transmission of $(26 \pm 3) \%$ is achieved with $\eta_{p}=(0.62 \pm 0.06) \%$, a value approximately half of that obtained for the same structure employing a conventional, reflective, and thick $\mathrm{Ag}$ cathode. A semitransparent tandem organic solar cell with $\eta_{p}=(0.48 \pm 0.02) \%$ and an average transmission of $(44 \pm 4) \%$ is also demonstrated. Semitransparent organic photovoltaic cells have potential uses as tinted and power-generating thin-film coatings on architectural surfaces, such as windows and walls. The use of a transparent top electrode also significantly simplifies the design of tandem cells, relaxing requirements for the placement of different absorbing materials at the maxima of optical fields introduced by reflective cathodes. ( ) 2006 American Institute of Physics. [DOI: 10.1063/1.2209176]
\end{abstract}

Small molecular weight bilayer heterojunction organic photovoltaic (OPV) cells are recognized for their possible uses in large-area, flexible, and low-cost power generation applications. The thicknesses of the OPV active layers are limited by the relatively short exciton diffusion lengths that are on the order of a few tens of nanometers. ${ }^{1}$ This leads to comparatively thin organic layers that can have a high optical transmissivity over the visible spectral range. Here, we demonstrate both single junction and tandem OPV cells that have a maximum average transmission of up to $(60 \pm 6) \%$ over the wavelength range from $\lambda=450$ to $750 \mathrm{~nm}$, coupled with a relatively high power conversion efficiency. These cells have the potential for use as power-generating tinted thin-film coatings on windows and other architectural surfaces. For example, they can be used to replace current, passive tinted window coatings with a large power-generating surface with nearly identical optical properties. Indeed, semitransparent windows using dye-sensitized $\mathrm{TiO}_{2}$ have already been demonstrated. ${ }^{2}$ Previous work in inorganic photovoltaics has achieved semitransparent solar cells by introducing small holes in crystalline ${ }^{3,4}$ and amorphous ${ }^{5} \mathrm{Si}$ cells, and by the use of two transparent conducting oxide electrodes in $\mathrm{Cu}(\mathrm{InGa}) \mathrm{Se}_{2}$ cells. ${ }^{6}$ Furthermore, ultrathin film organic solar cells have significant constraints on the placement of the absorbing layers with respect to the reflective metal cathode at the peak of the incident optical field. The use of transparent electrodes eliminates this constraint, thereby considerably simplifying the design of both single heterojunction and multijunction (tandem) cells.

The cells employ the archetype donor-acceptor (D-A) bilayer system ${ }^{7}$ comprised of copper phthalocyanine (CuPc) and 3,4,9,10-perylenetetracarboxylic bis-benzimidazole (PTCBI). Purified organic source materials ${ }^{8}$ were loaded into

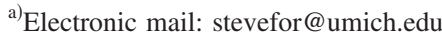

a high vacuum thermal evaporation chamber with a base pressure of $5 \times 10^{-7}$ Torr. The materials were deposited at a growth pressure of $1 \times 10^{-6}$ Torr, at a rate of $2 \AA / \mathrm{s}$. When not under high vacuum, the sources were stored under a nitrogen atmosphere, with water and oxygen levels $\leqslant 1 \mathrm{ppm}$. Glass substrates coated with a 1500 A thick indium tin oxide (ITO) anode having a sheet resistance of $15 \Omega / \square$ were cleaned as described elsewhere, ${ }^{8}$ followed by $5 \mathrm{~min}$ exposure to an ultraviolet-ozone surface treatment. The double heterojunction solar cell consisted of a $200 \AA$ thick $\mathrm{CuPc}$ donor layer, a $250 \AA$ thick PTCBI acceptor, and a $100 \AA$ thick bathocuproine (BCP) exciton blocking layer, ${ }^{9}$ while the two-cell tandem structure consisted of two, $200 \AA$ thick CuPc by $200 \AA$ thick PTCBI layer pairs forming heterojunctions separated by a sheet of $\sim 0.5 \mathrm{~nm}$ diameter $\mathrm{Ag}$ nanoparticles, ${ }^{10}$ followed by a $100 \AA$ A thick BCP layer. Layers of $\mathrm{Ag}$, ranging in thickness from 0 to $100 \AA$, were thermally deposited through a shadow mask with an array of $1 \mathrm{~mm}$ diameter holes, followed by a $350 \AA$ thick layer of ITO sputter deposited at $10 \mathrm{~W}$ and $13.56 \mathrm{MHz}$, resulting in a deposition rate of $5 \AA / \mathrm{min}$. A vacuum break and exposure to air between organic film and cathode deposition were necessary prior to attachment of the shadow mask, which was done in a nitrogen ambient. Solar cell performance was characterized in the dark and under simulated AM1.5G solar illumination (uncorrected for solar spectral mismatch) using a $150 \mathrm{~W}$ xenon arc lamp. ${ }^{11}$ Transmission measurements were obtained using a Perkin-Elmer spectrometer on unpatterned films grown simultaneously alongside of the photovoltaic structures.

Figure 1(a) shows a photograph of a metal-free organic thin-film photovoltaic cell with a patterned stripe of sputterdeposited ITO placed over a background image to demonstrate their transparent appearance. The stripe of ITO is not clearly discernible because of the similar transmissivity with 
(a)
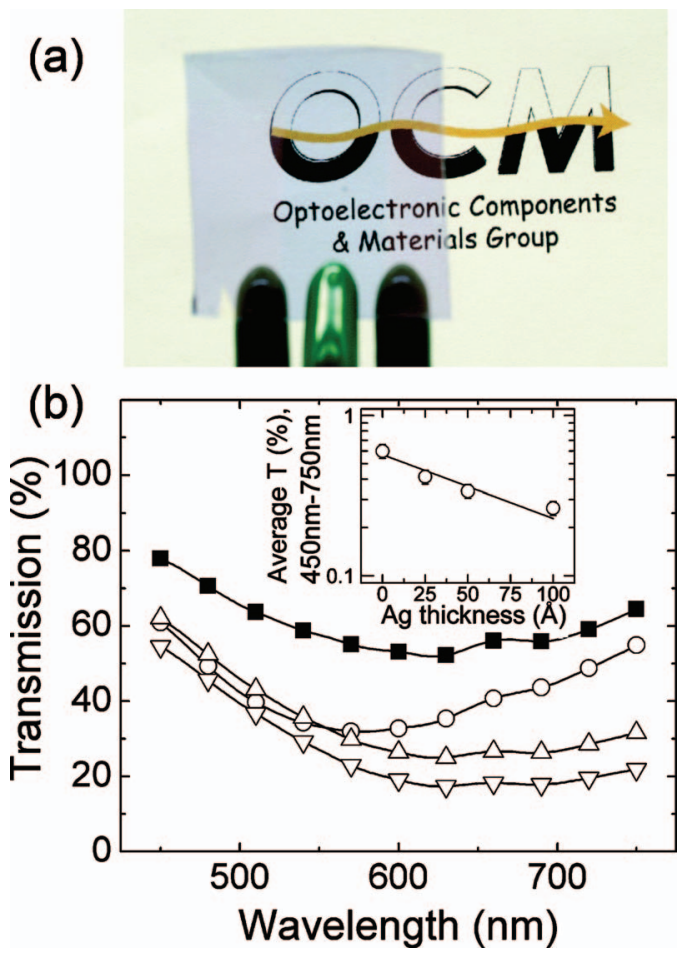

FIG. 1. (Color) (a) Image of a photovoltaic double heterojunction structure consisting of $200 \AA$ copper phthalocyanine/250 $\AA$ 3,4,9,10perylenetetracarboxylic bis-benzimidazole/100 $\AA$ bathocuproine on glass substrate precoated with an indium tin oxide (ITO) anode layer. The sample has a $350 \AA$ sputter-deposited vertical stripe ITO cathode, which is not discernible due to the similar transmissivity with and without the ITO cathode. (b) Transmission spectra for photovoltaic structures as in (a), with $0 \AA$ (filled squares), $25 \AA$ (open circles), $50 \AA$ (open triangles), and $100 \AA$ (open upside-down triangles) thick Ag cathode layers. The curve shapes reflect the absorption spectrum of copper phthalocyanine. The inset shows optical transmission averaged over wavelengths of $450-750 \mathrm{~nm}$ vs thickness of the Ag layer. The line represents a fit to the data.

$[(60 \pm 6) \%$ averaged over the overlap between the thin-film absorbance and the solar spectrum] and without $[(59 \pm 6) \%]$ the ITO cathode. The transmission spectra of four semitransparent PV cells on glass, with the $\mathrm{Ag}$ in the compound ITO/Ag cathode ranging in thickness from 0 to $100 \AA$, are shown in Fig. 1(b). For the metal-free structure, the transmission $T$ varies from approximately $60 \%$ at wavelengths between $\lambda=450$ and $700 \mathrm{~nm}$, with a minimum at $\lambda=625 \mathrm{~nm}$ corresponding to the absorption maximum of $\mathrm{CuPc} .{ }^{1} \mathrm{At} \mathrm{a} \mathrm{Ag}$ thickness of $25 \AA$, the spectrum takes a slightly different shape, with enhanced absorption occurring in the blue. We attribute this effect to the discontinuity of thin $\mathrm{Ag}$ layers, leading to surface-plasmon enhancement of the optical field in the PTCBI layer. ${ }^{10}$ The trend of decreasing $T$ with increasing Ag layer thickness is shown in the inset of Fig. 1(b). The best fit to $T=T_{o} e^{(-x / \delta)}$ (where $T_{o}$ is the average transmission of the metal-free device, $x$ is the Ag thickness, and $\delta$ is the average optical skin depth), plotted as a solid line in Fig. 1 (b), gives $\delta=(110 \pm 20) \AA$. This is similar to previous calculations of $\delta$ for thin $\mathrm{Mg}-\mathrm{Ag}$ layers used as electroninjecting contacts in transparent organic light-emitting devices. ${ }^{12}$

The power conversion efficiency, $\eta_{p}$, and the responsivity, $J_{\mathrm{sc}} / P_{o}$, are plotted versus Ag layer thickness in Fig. 2. Here, $J_{\mathrm{sc}}$ is the short circuit current density and $P_{o}$ is the incident optical power density. The dashed lines represent the values obtained for conventional (i.e., nontransparent)

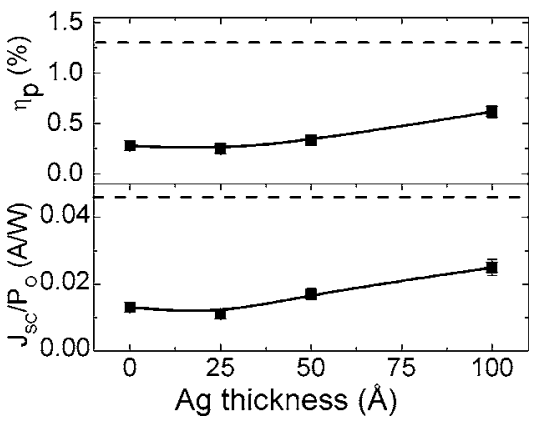

FIG. 2. Power conversion efficiency, $\eta_{p}$, and responsivity, $J_{\mathrm{sc}} / P_{o}$, under simulated AM1.5G solar illumination vs cathode Ag layer thickness. The solid lines serve as guides to the eye, while the dashed lines represent values for conventional devices.

devices and the solid lines connecting data points are a guide for the eye. Conventional devices with the same organic layer thicknesses employing a thick Ag cathode were grown alongside the semitransparent devices as experimental controls. The conventional devices exhibited $\eta_{p}=(1.3 \pm 0.1) \%$, whereas the Ag-free structure has $\eta_{p}=(0.28 \pm 0.03) \%$. Increasing the Ag layer thickness from 25 to $100 \AA$ results in $\eta_{p}=(0.25 \pm 0.03) \%$ to $(0.62 \pm 0.06) \%$, respectively.

Comparisons of the semitransparent cell efficiencies with that of the control must account for reflections that occur in the latter cell, contributing to the photocurrent via optical interference effects. ${ }^{1}$ The increase in power conversion efficiency with $\mathrm{Ag}$ thickness is primarily due to the increasing responsivities of the cells with increasing cathode reflectivity. Thus, the corresponding responsivities are $(0.013 \pm 0.001) \mathrm{A} / \mathrm{W}$ (for a Ag thickness of $x=0 \AA$ ), $(0.011 \pm 0.001) \mathrm{A} / \mathrm{W} \quad(x=25 \AA), \quad(0.017 \pm 0.002) \mathrm{A} / \mathrm{W}$ $(x=50 \AA), \quad(0.025 \pm 0.002) \mathrm{A} / \mathrm{W} \quad(x=100 \AA), \quad$ and $(0.046 \pm 0.003) \mathrm{A} / \mathrm{W}$ for the conventional, thick $\mathrm{Ag}$ cathode cell. Simulations of the optical field within the metal-free structure predict that $J_{\mathrm{sc}}$ should be only $28 \%$ of that obtained for a conventional $\mathrm{Ag}$ cathode cell, which is in agreement with the experimentally determined ratio of $0.28 \pm 0.04$.

The small difference in the open circuit voltages of $V_{\mathrm{oc}}=(0.40 \pm 0.02) \mathrm{V}$ for the semitransparent cells, and $(0.45 \pm 0.02) \mathrm{V}$ for the control, is expected due to the lower responsivity of the semitransparent cells. The open circuit voltage can be calculated using $V_{\text {oc }} \approx(n k T / q) \ln \left(J_{\mathrm{sc}} / J_{s}+1\right)$, where $n$ is the ideality factor, $q$ is the electron charge, $k T$ is the thermal energy at temperature $T$, and $J_{s}$ is the reverse saturation current density. ${ }^{13}$ Using typical values for our conventional cells $\left(n=1.64, J_{s}=10^{-4} \mathrm{~A} / \mathrm{cm}^{2}\right)$, we calculate $V_{\text {oc }}=0.46 \mathrm{~V}$ at $T=300 \mathrm{~K}$, and $0.40 \mathrm{~V}$ for the lowest responsivity transparent cell, values in agreement with the experimental results. All semitransparent cells have a fill factor of $\mathrm{FF}=0.55 \pm 0.09$, which is somewhat less than for the control (where $\mathrm{FF}=0.62 \pm 0.05$ ), possibly due to resistance of the compound cathode, and reduced $J_{\mathrm{sc}}$ and $V_{\mathrm{oc}}$.

External quantum efficiency (EQE) data are shown in Fig. 3 for both conventional and semitransparent cells, with the latter having having $x=0,25,50$, and $100 \AA \mathrm{Ag}(x) / \mathrm{ITO}$ cathodes. The curves all have similar shapes, with a peak EQE near $\lambda=625 \mathrm{~nm}$ of $(17 \pm 1) \%$ for the conventional device, and $(5 \pm 1) \%,(4 \pm 1) \%,(6 \pm 1) \%$, and $(10 \pm 1) \%$ for the transparent cells, respectively. These data are consistent with 


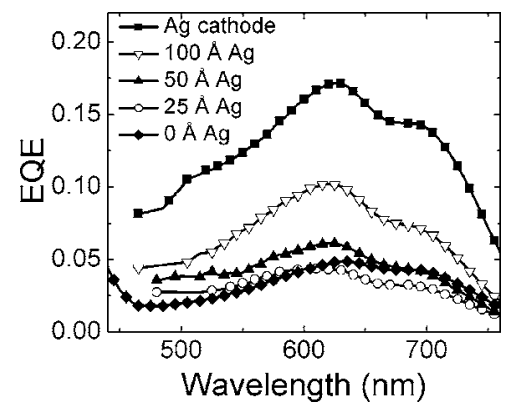

FIG. 3. External quantum efficiency (EQE) for a conventional, opaque cell (filled squares), a semitransparent cell with a $100 \AA$ thick Ag/ITO compound cathode (open upside-down triangles), a semitransparent cell with a $50 \AA$ thick Ag/ITO compound cathode (filled triangles), a semitransparent cell with a $25 \AA$ thick Ag/ITO compound cathode (open circles), and a semitransparent cell with an ITO-only cathode (filled diamonds).

the expectations developed by analyzing the behavior of responsivity with increasing Ag thickness.

One possible advantage of the semitransparent structure is the possibility of stacking many cells to achieve even higher efficiency. We note that optical interference effects constrain the design of tandem cells when the top metal cathode is highly reflective. That is, because there is little reflection at the cathode in the semitransparent cells, optical interference effects between the incident and reflected optical fields are reduced, yielding a more uniform intensity distribution throughout the active layer structure. This relaxes the conditions restricting the placement of absorbing layers at a particular distance from the reflecting surface to maximize cell responsivity.

Current-voltage characteristics under AM1.5G, 1 sun intensity simulated illumination of a single, metal-free cell and a metal-free tandem cell are shown in Fig. 4. The single cell has $T=(60 \pm 6) \%, \quad \eta_{p}=(0.28 \pm 0.02) \%, \quad V_{\mathrm{oc}}=0.38 \mathrm{~V}$, $\mathrm{FF}=0.54$, and $J_{\mathrm{sc}} / P_{o}=0.014 \mathrm{~A} / \mathrm{W}$, while the tandem cell has $T=(44 \pm 4) \%, \quad \eta_{p}=(0.48 \pm 0.02) \%, \quad V_{\mathrm{oc}}=0.75 \mathrm{~V}, \quad \mathrm{FF}=0.49$,

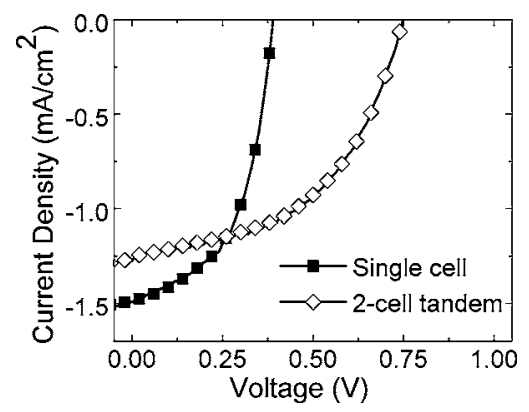

FIG. 4. Current-voltage characteristics for a single metal-free heterojunction cell (filled squares), and a two-cell tandem stack (open diamonds). and $J_{\mathrm{sc}} / P_{o}=0.013 \mathrm{~A} / \mathrm{W}$. This represents an expected doubling in $V_{\mathrm{oc}}$ and a near doubling in $\eta_{p}$ for the tandem cell. This tandem structure has not been fully optimized and we anticipate that even higher efficiencies can be achieved and a larger number of stacks implemented using this concept.

In conclusion, we have demonstrated a semitransparent small molecular weight organic photovoltaic cell with a peak average transmission over the visible spectrum of $(60 \pm 6) \%$, and a power conversion efficiency of $\eta_{p}=(0.28 \pm 0.03) \%$ under simulated AM1.5G, 1 sun illumination. Our results indicate that the exciton separation and charge extraction efficiencies of transparent devices are equivalent to those obtained for a conventional organic photovoltaic cell employing the same materials combinations, once the different optical characteristics of the two cell structures are taken into consideration. In addition, we have demonstrated a semitransparent tandem photovoltaic cell with nearly double the power conversion efficiency and open circuit voltage of a similar, single element cell. These results suggest that semitransparent organic photovoltaic cells have application to tinted power-generating and light attenuating coatings for windows and other architectural surfaces. Furthermore, the elimination of the reflective cathode considerably simplifies the design of single and multiple junction OPV cells.

This work was supported in part by funds from the Air Force Office of Scientific Research, the National Renewable Energy Laboratory (NREL), and Global Photonic Energy Corp., Ewing, NJ.

${ }^{1}$ P. Peumans, A. Yakimov, and S. R. Forrest, Appl. Phys. Rev. 93, 3693 (2003).

${ }^{2}$ M. G. Kang, N. Park, Y. J. Park, K. S. Ryu, and S. H. Chang, Sol. Energy Mater. Sol. Cells 75, 475 (2003).

${ }^{3}$ R. Kühn, A. Boueke, A. Kress, P. Fath, G. P. Willeke, and E. Bucher, IEEE Trans. Electron Devices 46, 2013 (1999).

${ }^{4}$ P. Fath, H. Nussbaumer, and R. Burkhardt, Sol. Energy Mater. Sol. Cells 74, 127 (2002).

${ }^{5}$ A. Takeoka, S. Kouzuma, H. Tanaka, H. Inoue, K. Murata, M. Morizane, N. Nakamura, H. Nishiwaki, M. Ohnishi, S. Nakano, and Y. Kuwano, Sol. Energy Mater. Sol. Cells 29, 243 (1993).

${ }^{6}$ T. Nakada, Y. Hirabayashi, T. Tokado, D. Ohmori, and T. Mise, Sol. Energy 77, 739 (2004).

${ }^{7}$ C. W. Tang, Appl. Phys. Lett. 48, 183 (1986).

${ }^{8}$ R. F. Salzman, J. Xue, B. P. Rand, A. Alexander, M. E. Thompson, and S. R. Forrest, Org. Electron. 6, 242 (2005).

${ }^{9}$ P. Peumans, V. Bulovic, and S. R. Forrest, Appl. Phys. Lett. 76, 2650 (2000).

${ }^{10}$ B. P. Rand, P. Peumans, and S. R. Forrest, J. Appl. Phys. 96, 7519 (2004).

${ }^{11}$ Oriel Instruments, 150 Long Beach Blvd., Stratford, CT 06615.

${ }^{12}$ V. Bulovic, G. Gu, P. E. Burrows, S. R. Forrest, and M. E. Thompson, Nature (London) 380, 29 (1996).

${ }^{13}$ J. Xue, S. Uchida, B. P. Rand, and S. R. Forrest, Appl. Phys. Lett. 84, 3013 (2004). 